\title{
The coronavirus spread: the Italian case
}

\author{
Aldo Bonasera ${ }^{1,2, a}{ }_{\mathbb{D}}$, G. Bonasera ${ }^{1}$, Suyalatu Zhang $^{3}$ \\ ${ }^{1}$ Cyclotron Institute, Texas A\&M University, College Station, TX 77843, USA \\ 2 LNS-INFN, v. Santa Sofia, 64-95123 Catania, Italy \\ 3 College of Mathematics and Physics, Inner Mongolia University for Nationalities West, 536 Huolinhe Rd., \\ Tongliao 028000, Inner Mongolia, China
}

Received: 13 April 2020 / Accepted: 25 May 2020 / Published online: 1 June 2020

(C) Società Italiana di Fisica and Springer-Verlag GmbH Germany, part of Springer Nature 2020

\begin{abstract}
A model based on population growth, chaotic maps, and turbulent flows is applied to the spread of Coronavirus for each Italian region in order to obtain useful information and help to contrast it. We divide the regions into different risk categories and discuss anomalies. The worst cases are confined between the Appenine and the Alps mountain ranges but the situation seem to improve closer to the sea. The Veneto region gave the most efficient response so far and some of their resources could be diverted to other regions, in particular, more tests to the Lombardia, Liguria, Piemonte, Marche and V. Aosta regions, which seem to be worst affected. We noticed worrying anomalies in the Lazio, Campania and Sicilia regions to be monitored. We stress that the number of fatalities we predicted on March 12 has been confirmed daily by the bulletins. This suggests a change of strategy in order to reduce such number maybe moving the weaker population (and negative to the virus test) to beach resorts, which should be empty presently. The ratio deceased/positives on April 4, 2020 is $5.4 \%$ worldwide, $12.3 \%$ in Italy, $1.4 \%$ in Germany, $2.7 \%$ in the USA, $10.3 \%$ in the UK and $4.1 \%$ in China. These large fluctuations should be investigated starting from the Italian regions, which show similar large fluctuations.
\end{abstract}

\section{Introduction}

The 2020 widespread of the Coronavirus or COVID-19 virus could be compared to the spread of the Red Weevil (Rhynchophorus Ferrugineus) in the Mediterranean or fires in California. They start in one or more localized places and quickly spread over larger and larger regions until it becomes difficult to stop them. After that the spread continues to 'affect' more and more regions until there is some 'fuel', i.e., palm trees for the Red Weevil or woods for the fire. This mechanism is similar to physical systems, for instance turbulent flow or chaotic maps [1-7], where a small perturbation grows exponentially and then saturates to a finite value. These at first sht different systems have some common features: a small perturbation, which we will indicate as $\mathrm{d}_{0}$, grows exponentially with a coefficient $\gamma$, the Lyapunov exponent, and finally saturates [1-3] to a value $d_{\infty} \gg \mathrm{d}_{0}$. The fact that every chaotic system saturates to a finite value, even though might be very large, indicates that the 'phase-space' is, however, limited and reflects some conservation laws, such as energy conservation for a physical

\footnotetext{
a e-mail: abonasera@comp.tamu.edu (corresponding author)
} 


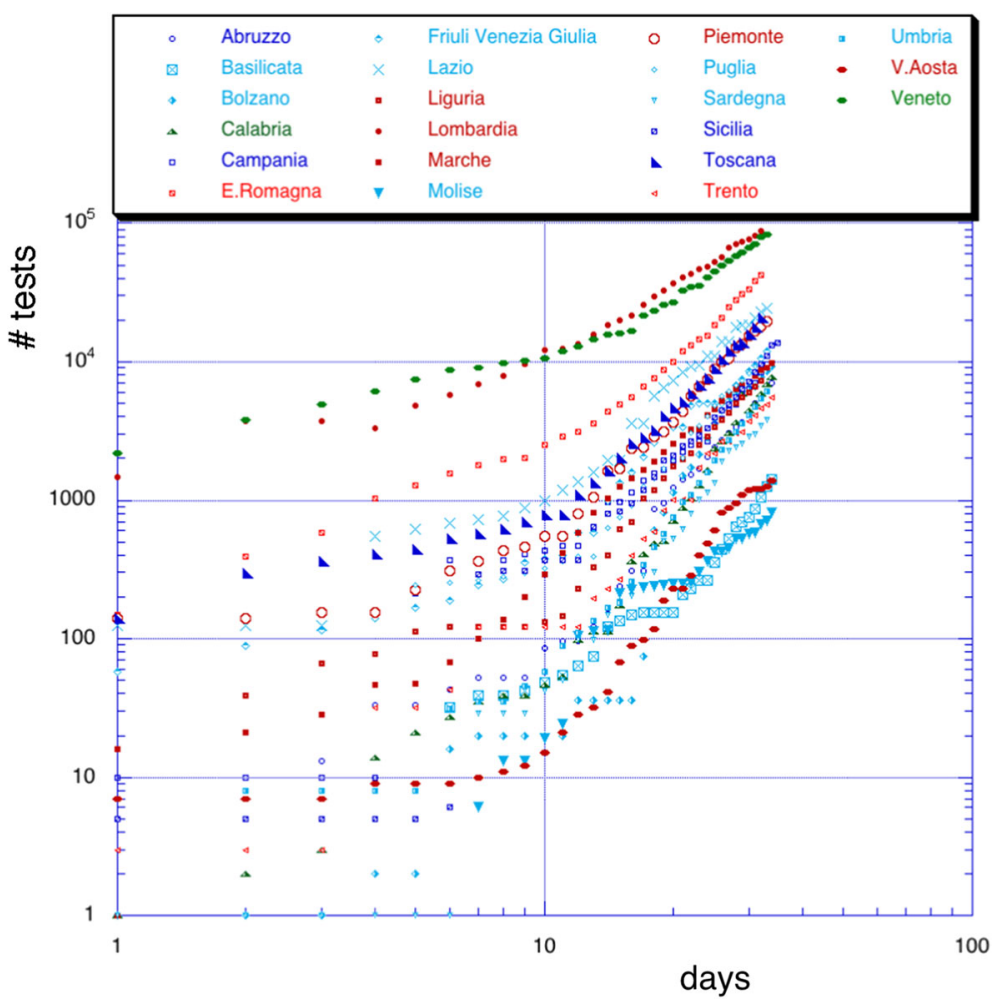

Fig. 1 Number of tests as function of the day, starting from February 24, 2020. The different Italian regions are indicated in the inset

system or the number of palm trees for the Red Weevil. We can write the number of people for instance positives to the virus (or deceased for the same reason) as:

$$
N(d)=\frac{d_{0} d_{\infty}}{d_{0}+d_{\infty} e^{-\gamma d}} .
$$

In the equation, $d$ gives the time, in days, from the starting of the epidemic, or the time from the beginning of the tests to isolate the virus. At time $d=0, N(0) \approx d_{0}$ which is the very small value (or group of people) from which the infection started. In the opposite limit, $d \rightarrow \infty, N(\infty)=d_{\infty}$, the final number of affected people by the virus. Equation (1) is the logistic equation first introduced by Verhulst [8] as a model of population growth. It is the solution of a simple first-order non-linear ordinary differential equation. In Ref. [2], it was introduced to study the transition to turbulent flow, Eq. $(26.7)^{2}$. It is also the continuous version of the logistic map [1, 4]. In a recent paper [7], we have analyzed the 2003 SARS and the COVID-19 viruses using the equation written above and fitting the three parameters to the data. Despite its simplicity (for instance, it lacks a proper accounting of the spatial spreading of the pandemics), the model reproduces the data very well on a daily basis starting from March 12 for the Italy case [7]. This might be coincidental but it is further supported by the analysis of the virus spread in other countries [7].

The first important result that we pointed out is that to have information on the number of positive to the virus (or fatalities) is not statistically relevant if we do not know the total 


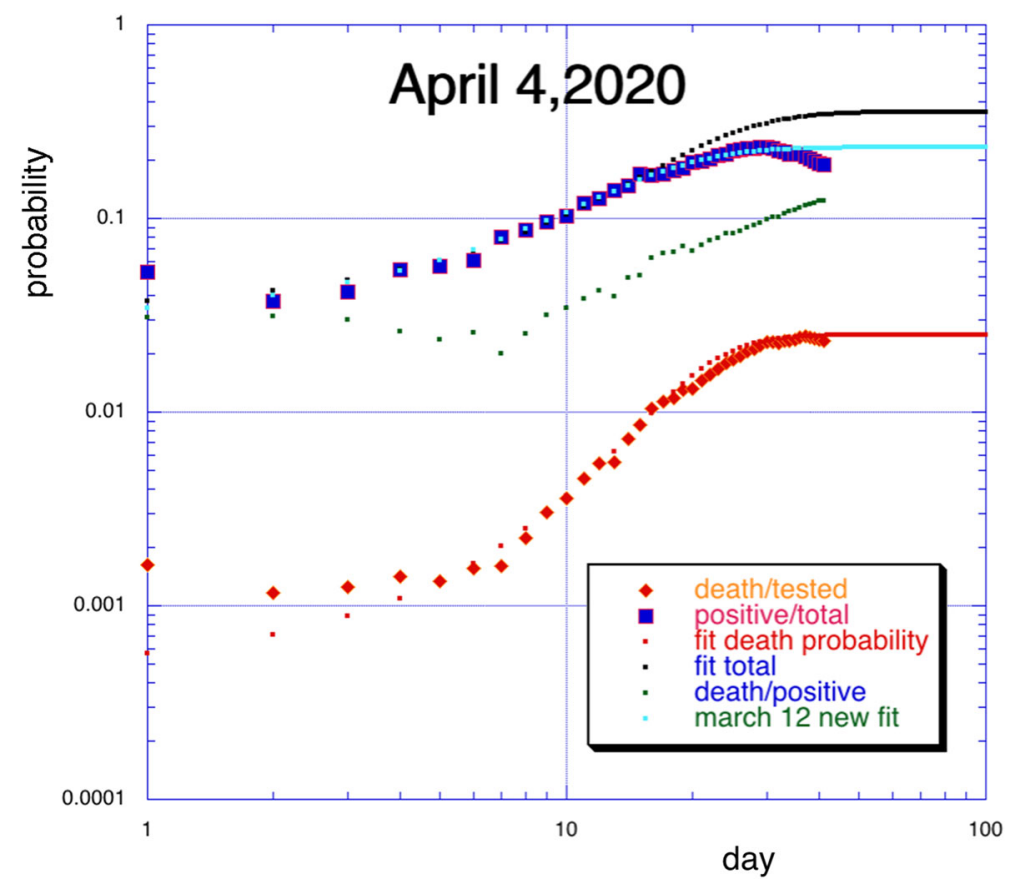

Fig. 2 Italy: number of positives to the test (upper points) and number of fatalities (lower points) both normalized by the total number of tests performed as a function of the day from February 24, 2020. The green full squares in the middle give the ratio fatalities/positive. The curves are the results of the fits using Eq. (1). The fits were performed before March 12, 2020 while the actual data has been updated daily to April 4, 2020

number of tests performed each day and possibly the method chosen to perform the tests. The method to choose the people to be tested might be biased because of the large number of people affected and the limited amount of tests and facilities. We have been able to obtain quickly the total number of daily tests (and other relevant quantities) from https://github. com/pcm-dpc/COVID-19, but this is not always possible for other countries.

In Fig. 1, we plot the total number of tests performed in all Italian regions starting from February 24,2020 . The different regions are indicated by different colors and/or symbols. The reason for each color will be discussed more in detail later and we have divided them into the dark red, red, blue, cyan, and green colors depending on the probability to find a positive to the virus in that region. So dark red gives the highest probability while green is the lowest. Notice that the Lombardy, Veneto and E. Romagna regions performed the most test. The other important feature to notice is the change of slope after day 10 (starting from February 24,2020), which means that after that day the number of tests performed daily more than doubled. This change on the number of tests and the different number for different countries or regions make it difficult to make predictions on absolute values such as the total number of positives to the virus or any other quantities. Thus it is statistically more relevant to define probabilities from the ratio of positives divided by the total number of tests or the number of fatalities divided by the total number of tests, etc.

Equation (1) is well suited to predict these probabilities once a fit has been performed on some preliminary results, we apply it first to Italy as a whole. In the Fig. 2, we show the results of the fit by fixing the parameters on March 12, 2020. A previous fit after the start of 


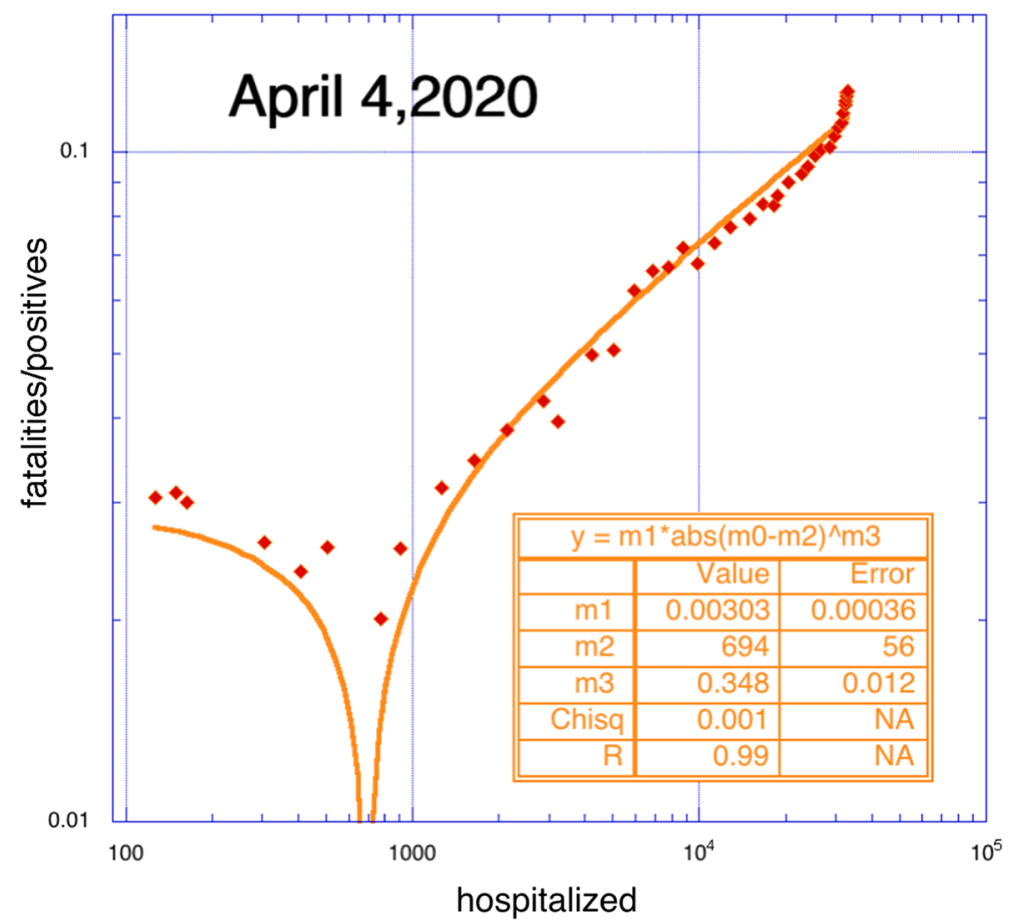

Fig. 3 Ratio fatalities/positives as function of the people hospitalized daily. The full line is the result of the fit given by Eq. (2). The fit values are given in the inset

the epidemic was performed on the positives, it is given by the upper (black) points in the figure. As we saw in Fig. 1 after about 10 days the number of tests was more than doubled and also quarantine measures were taken by the Italian government. This led to the new fit on March 12 given by the cyan points in the figure. Since then we have not modified the fit but just added the new daily points which seem to follow the new fit for the positives up to April 4, 2020. As we can see there seem to be a decrease for the last points. A decrease from the prediction means that the probability to be positive to the virus is decreasing and social distancing plus other measures are producing good results. If we now analyze the fatalities, we first of all notice that the original prediction is followed by the data and we did not need to perform a new fit on March 12 at variance with the number of positives. Furthermore, the probability for fatalities seems to follow the prediction and little or no decrease is observed. Another important quantity plotted is the ratio deceased/positives, which should be somewhat independent on the total number of tests if the method to choose the people to be tested does not change. As we can see the ratio keeps increasing and becomes larger than $10 \%$. This is much higher than Germany for instance, which has a large number of positives as well and can be statistically compared to Italy. Other countries like Spain and UK show similar values for the ratio like Italy. These large fluctuations might be attributed to the different health facilities, ventilators, hospital overcrowding, etc. It is important to coordinate the action in different countries to try to understand the reason for the discrepancies and save many lives.

One possibility for the large number of fatalities in Italy respect to other countries could be a time delay between being tested positive and passing away. In fact, in Fig. 2, we notice that the ratio is less than $3 \%$ before day 10, similar to other countries. However, China, Germany, 


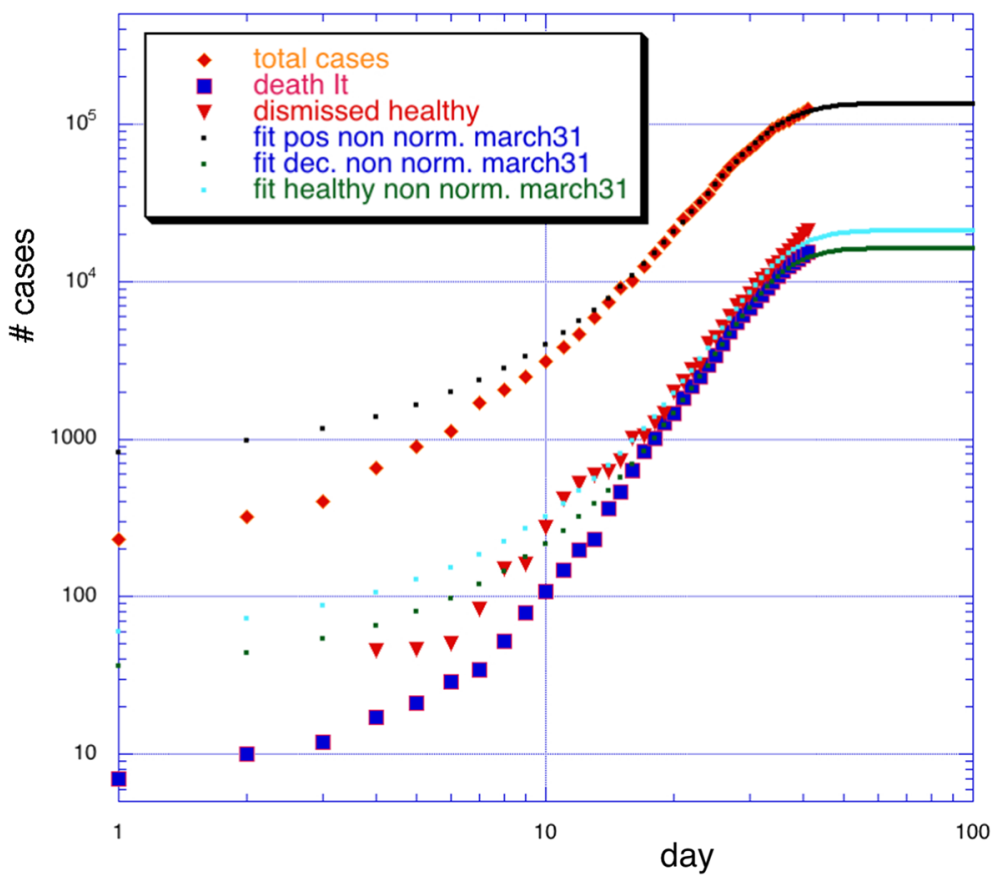

Fig. 4 Total number of positives, dismissed healthy and fatalities as function of the day. The fits using Eq. (1) were performed on March 31 and new data have been added daily

Spain, UK, and other countries have been contrasting the virus for more than 10 days already thus any transient effect should be finished. Another reason we could explore is hospital overcrowding which leads to a lack of resources for the emergency. In Fig. 3, we plot the quantity $\mathrm{y}=$ fatalities/positives as function of the number of people hospitalized each day. A clear correlation between the two variables is visible and we have parameterized it as:

$$
y(m 0)=m 1|m 0-m 2|^{m 3}
$$

$m_{1}, m 2$ and $m 3$ are fitting parameters and are displayed in the figure. In particular, $m 2$ gives a 'critical' value where the ratio grows quickly. This parameterization is inspired by critical phenomena such as the liquid-gas (second-order) phase transition in normal fluids and also in chaotic maps [1]. If taken literally this result would imply that hospitals should not admit more than $694 \pm 56$ people daily, however as discussed above for the total number of tests, this plot would be more meaningful if we would know how many patients can the hospital accommodate in normal and safe conditions. In other words, the number of hospitalized people might increase together with the number of hospitals involved thus to have an unequivocal correlation the total hospital capability should be known. The latter is not given in https://github.com/pcm-dpc/COVID-19 and, we hope, this will be addressed soon, together with the total number of ventilators available.

In any case we expect that some kind of correlation like in Fig. 3 will remain. Other delocalized facilities should be organized to do a preliminary screening and admit to the hospital only the most severe cases, below the capability limits for each facility. Figure 2 shows that at most 1 person out of 5 tests positive to the virus but being admitted in an overcrowded hospital increases the probability of serious complications, i.e., more than 1 


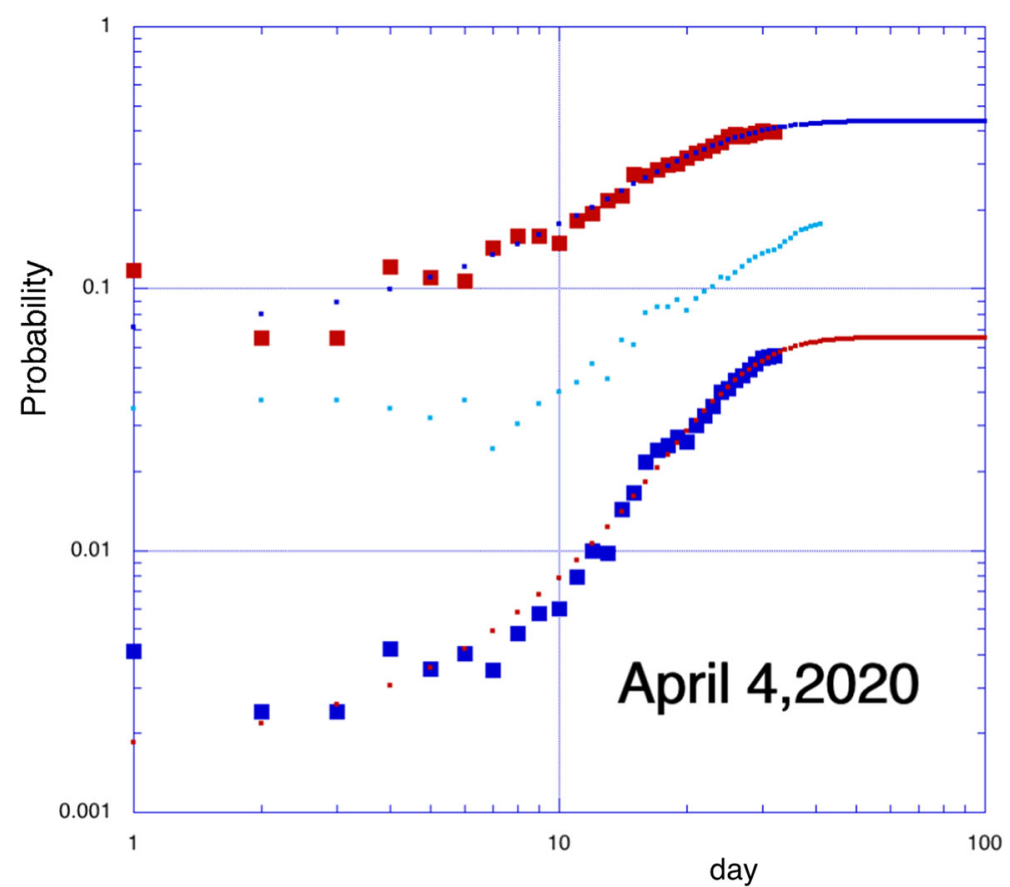

Fig. 5 Same as Fig. 2, for the Lombardy region. The fits were performed on March 26 but the data has been update to April 4,2020

person out of 50 might die (in Germany it is roughly 1 out of 300 presently). An overcrowded hospital implies that the number of ventilators (which seem to be the last resource to fight the virus) is not sufficient.

Equation (1) gives a very good description of the probability of being infected by the virus but does not give us any hint on when the virus will stop its deadly action. We could in principle apply the same equation to the number of positives for instance without normalizing by the total number of tests. We have shown that this procedure might be meaningless if the number of tests performed daily changes or varies from region to region. However, from Fig. 1 we notice that after day 10 the test number is a straight line, which implies that it is constant. Thus we may hope that Eq. (1) includes the trivial increase of daily tests in the three parameters and try to make predictions. This rather empirical method to predict the evolution of the spread might be justified only by the results. Once we get some confidence for some cases we can apply it to other cases keeping in mind that the total number of tests daily must be constant.

In the Fig. 4, we plot the total number of positives, dismissed healthy and fatalities as a function of the day for the Italian case. As we can see the fit reproduces rather well the behavior at longer times and the data seem to saturate. For shorter times (below day 10), the model disagrees with the data due to the fact that the number of tests performed daily increased. Thus the prediction of Fig. 4 should be taken with caution and we hope that at least the order of magnitude is correct. Refitting on April 5, 2020 increased the predictions to $1.43 \mathrm{e} 5 \pm 1625(1.35 \mathrm{e} 5), 17,904 \pm 296(16,162)$ and $28,556 \pm 889(20,954)$ for positives, fatalities and dismissed respectively (in parentheses the values of the fit on March 31, 2020). The values of the fits performed at different times are different which suggests that the model 


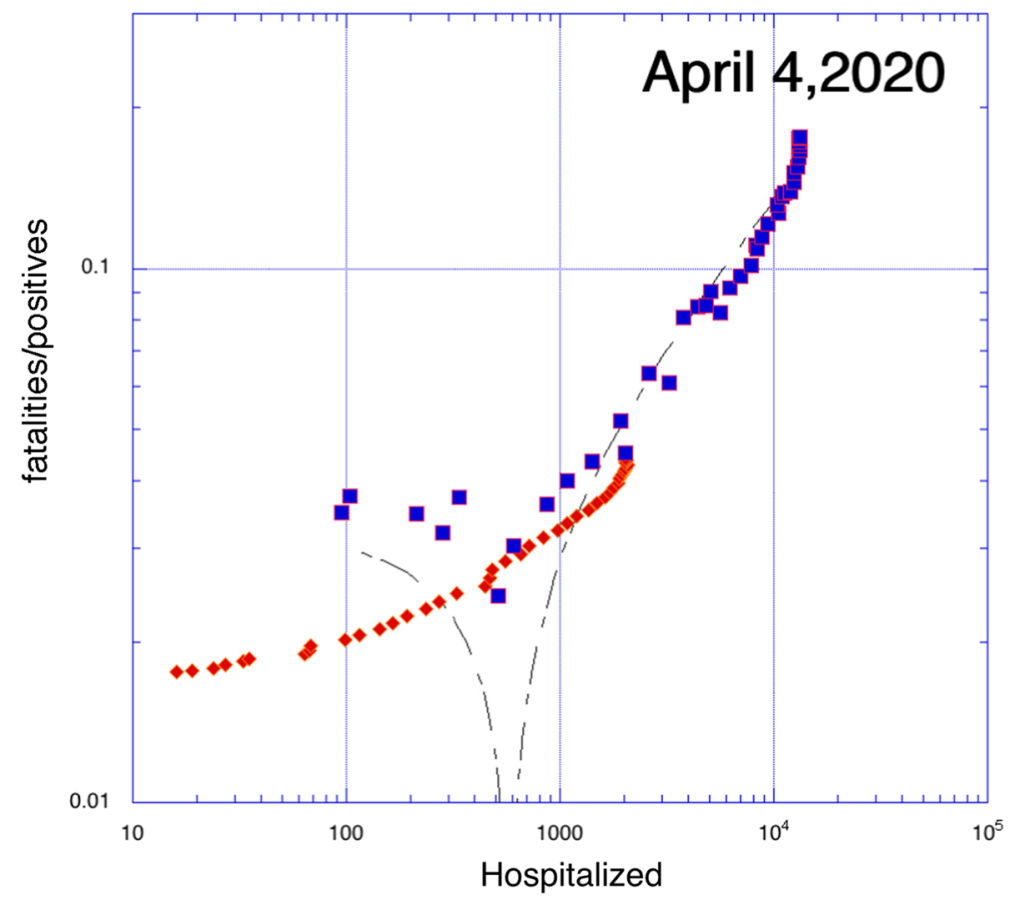

Fig. 6 Same as Fig. 3, for the Lombardy (full squares) and Veneto (full rhombs) regions

is better suited to predict the initial spread of the virus. A generalization to a two-step model able to predict longer time behavior is in preparation [9].

\section{Specializing the model to the lombardy region}

We can specialize the previous results to each Italian region to get important information on the spreading and also to unveil anomalies, which could indicate new centers for the epidemic. We start with the Lombardy region, the most affected by the pandemic.

In Fig. 5, we plot the probabilities as function of the day similar to the Italy case reported in Fig. 2. The probability to be infected reaches almost $50 \%$ while the fatalities are up to about $7 \%$ and the ratio fatalities/positives is almost $20 \%$, confirming, even if not needed, that Lombardy is the most affected region. Differently from Fig. 2 where a small decrease is observed at later days, Lombardy does not show any decrease but it seems very close to saturation. These results must be clarified since if this probability would reflect the actual population then 1 person out of 2 carries the virus. This is not the case since the method to choose the people to be tested is biased, i.e., those who show strong signs of infections are tested until there are no more tests available. This is one reason why some resources should be diverted temporarily to the Lombardy region starting for instance from the Veneto region; see Fig. 1.

The large number of fatalities may be due to hospital overcrowding and lack of equipment notably ventilators which seem to be the best tool to fight the infection or at least give more time to the organism to produce antibodies. Following the previous result for the 


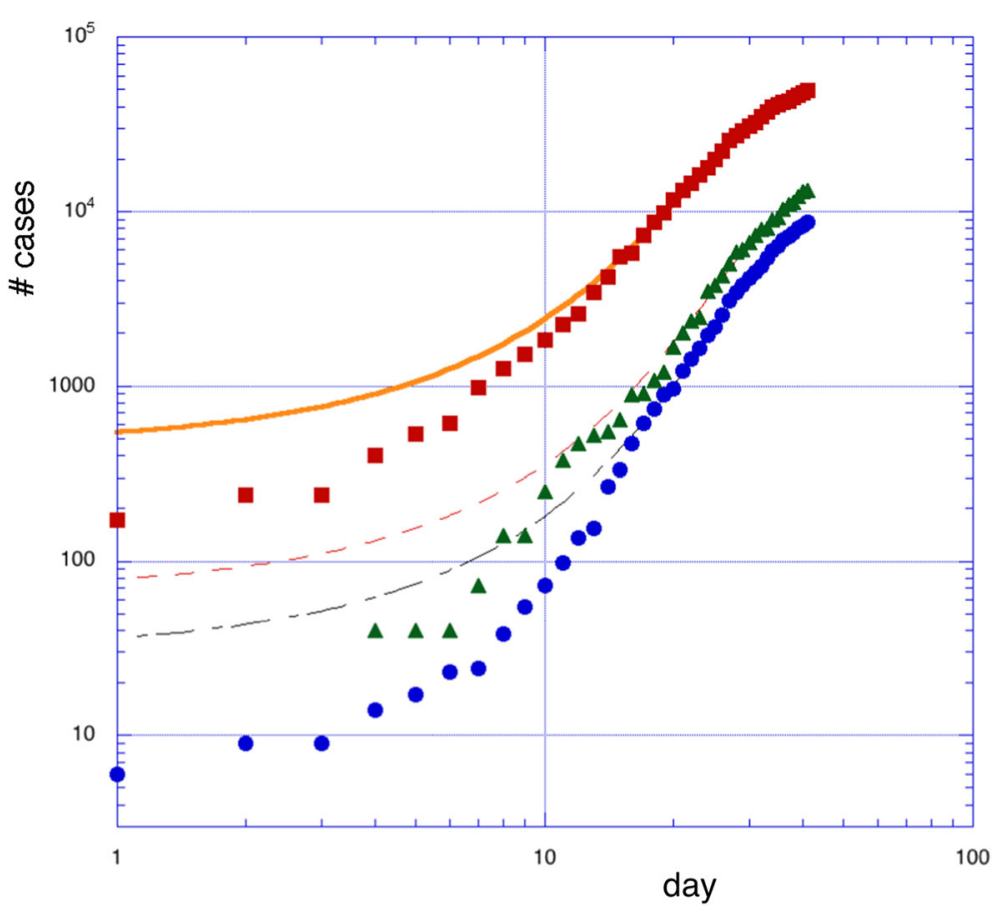

Fig. 7 Same as Fig. 4 for the Lombardy region. Full squares, triangles, and circles refer to positive, healthy and deceased cases, respectively. The fits are performed using Eq. 1

Italian case, we plot in Fig. 6 the ratio $y=$ fatalities/positives as function of the number of people hospitalized each day for the Lombardy and the Veneto regions. The behavior and the fit indicated in the figure confirm that Lombardy is the region contributing mostly to the epidemics. The smaller ratio for the Veneto region is consistent with the lower number of hospitalized persons but higher than other nations like Germany.

In Fig. 7, we use Eq. (1) to predict the total number of people affected by the virus. The fits on April 42,020, gave 53,286 $\pm 596,10,310 \pm 174$, and 15,943 \pm 501 for positives, fatalities, and dismissed cases respectively. These numbers can be compared to the National case given in the previous section: they contribute more than 50\%. Again the fits are not so good at shorter times due to the changing number of tests performed daily.

\section{All regions}

Following the methods outlined in the previous section, we can summarize the results for each region by looking in detail to all the fits.

In Fig. 8 we plot the probability of being tested positive as function of time for all the Italian regions. The fits were performed using Eq. 1 and following the method explained in the previous sections. All the fits were performed on March 31, 2020. Different regions were grouped with different colors and symbols in the figure in order to distinguish them according to the probability. The regions with the highest probability are Lombardy, Liguria, Piemonte, Marche and V.Aosta. Some might come as a surprise but recall that we are plotting 


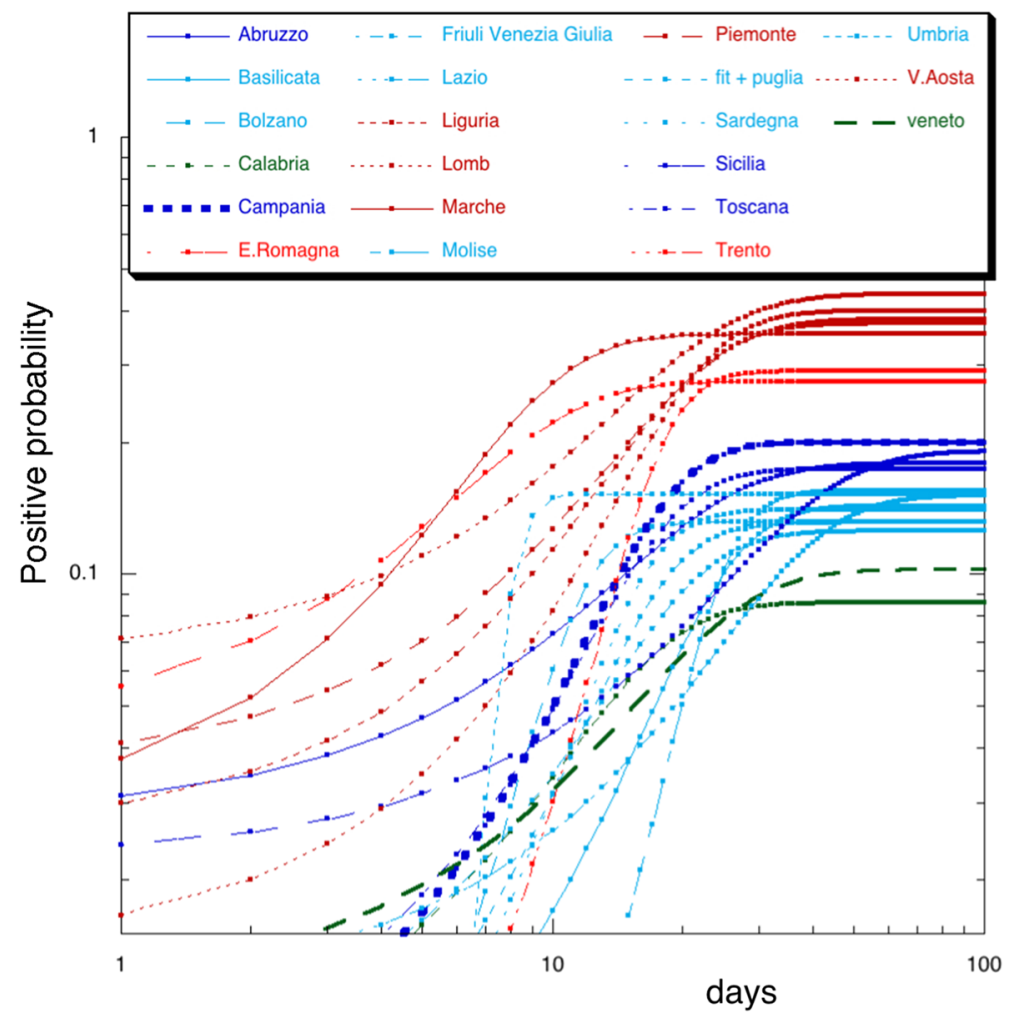

Fig. 8 Probability of testing positive to the virus as function of time, from Eq. (1). Different regions are grouped by different colors

the positives divided by the total number of tests. The Veneto region, which is one of the most affected, is represented by the green color, one of the lowest probabilities. This is due to the high number of tests performed in that region as shown in Fig. 1. Comparing Figs. 1 and 8 one could find reasons to shift resources as needed. As a preliminary method, we should shift resources from regions, which have less than $15 \%$ probability (below the cyan color in the figure) to be infected to higher probability regions. This could be done on a temporary basis, say for a week to see if the probabilities, for the most affected regions, decrease. Of course, ideally to increase the total number of tests everywhere would be the best solution. A probability say of $30 \%$ means that almost 1 person out of 3 is affected, thus even in apartments with more than 3 persons living in it, social distancing and other precautions should be enforced. This virus might be asymptomatic, i.e., we might carry it and show no signs, thus the importance to obtain better estimates of the probabilities.

It is also important to notice that some regions reach the saturation value earlier than others. For instance, the Marche (dark red symbols) and E. Romagna (red symbols) regions saturate earlier than their respective color groups. A faster saturation might not be good because it might give not enough time to the hospitals to deal with large number of patients arriving at the same time. The Umbria region (cyan symbols) is the one that saturates first 


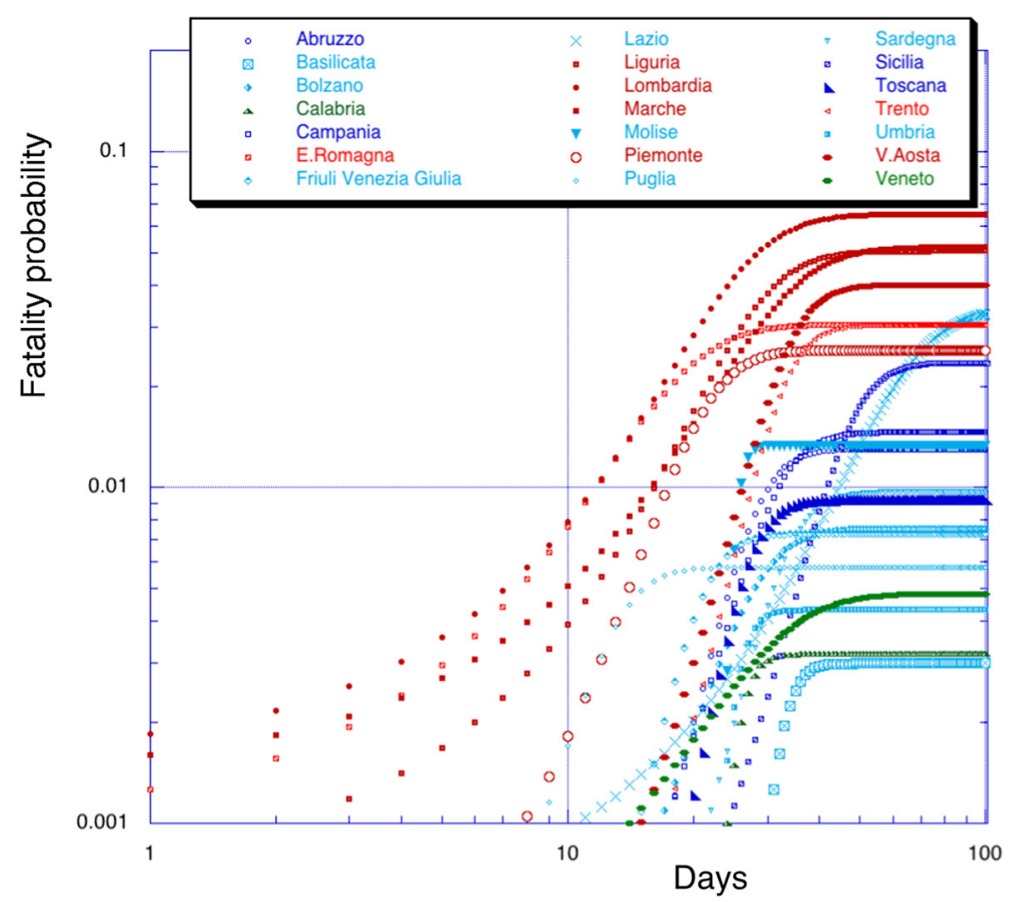

Fig. 9 Fatalities (probability) as function of time for all the Italian regions. Notice the switch of color ordering for some regions compared to Fig. 8

fortunately with a small number of positives and a large number of tests. For reference the same probability as in Fig. 8 for Germany is about 10\%, https://www.worldometers.info/ coronavirus/\#countries, which would be the first goal: perform enough tests to be sure that all regions are effectively below $10 \%$ probability.

In Fig. 9, we plot the fatality probability as function of time. Notice that the color grouping is not maintained and in particular we notice the large 'jump' of the Lazio region (cyan symbols) which goes 'three colors up', while there is some 'improvement' for Piedmont (dark red symbols). The Veneto region, which is very close to the pandemic center (Lombardy), has a fatality rate less than $0.5 \%$. This is the minimum goal, which can be reached by many other regions improving on the support system and maybe moving resources around. The reason(s) why the Lazio region is performing so poorly is not clear. The 'best performing' regions like Calabria, Basilicata and Umbria are located in the center, southern part of Italy, far away from the center of the infection [7]. Sicily is also a surprise being the most southern region and having such a large fatality rate, $2.2 \%$. For comparison, the probability for Germany is less than $0.2 \%$.

In Fig. 10, we plot the ratio fatalities/positives, a quantity we have discussed the in previous sections. We mentioned the fact that such a ratio is less than $2 \%$ for Germany on April 5,2020 a value similar to the Basilicata region only, but while Germany had 100,000 positives and 1600 deaths, Basilicata had 278 and 13 cases respectively. 


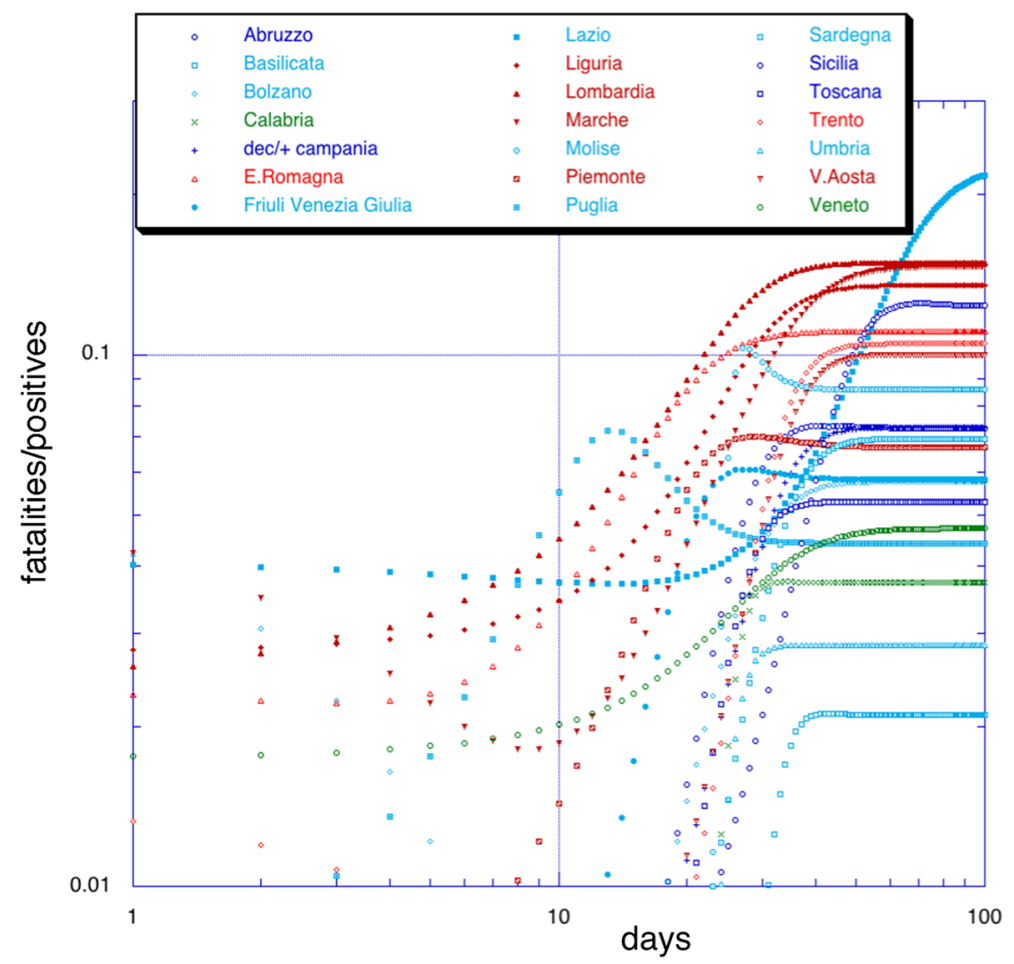

Fig. 10 Fatalities/positives versus time, see the previous figures for the color codes. For comparison, the same ratio for Germany was $1.6 \%$ on April 5, 2020

\section{Summary}

We investigated in detail the spread of COVID-19 in each Italian region. The overall statistics show that the spread is slowing down but not in some regions like Lombardy. The most negative feature is the statistical large number of fatalities as compared to the number of people tested positive to the virus. This is most probably due to hospital overcrowding and lack of enough tools like ventilators and sufficient personal protection equipment especially for the medical staff, which is in the front line. We cannot exclude that hospitals are a possible source of the infection, maybe the use of other public buildings transformed into temporary hospitals might help. Moving higher risk people, still negative to the virus, to lower density places like beach resorts might help. It is especially important in our opinion to put the different resources of the regions together to understand the spread more effectively. For instance, regions with a lower probability of infection (less than 15\%) might send some of their testing equipment, unused ventilators and maybe some medical personnel to higher risk regions on a temporary basis. Ventilators and personal protection equipment are the most needed tools. The country of cars, as well as electronics and fashion, might divert some industrial capabilities to fulfill the emergency in short times. The more we wait the more people dies. 


\section{References}

1. H.G. Schuster, Deterministic Chaos, VCH, 1995

2. L. D. Landau and E.M. Lifshitz, Fluid Mechanics, Elsevier, 2004

3. P. Arena, A. Bonasera, C. Brigante, M. Bucolo, F. Di Grazia, D. Lombardo, F. Sapuppo, and M. C. Virzí, in 2008 Mediterr. Conf. Control Autom. - Conf. Proceedings, MED'08 (2008)

4. V. Baran et al., Rom. J. Phys. 60, 1263 (2015)

5. S. Zhang et al., Phys. Rev. C 99, 044605 (2019)

6. A. Bonasera et al., Rivista Nuovo Cimento, 23(2):1 (2000)

7. A. Bonasera, S. Zhang, Front. Phys. 8, 171 (2000). https://doi.org/10.3389/fphy.2020.00171

8. P.-F. Verhulst, Notice sur la loi que la population poursuit dans son accroissement. Correspondance Mathématique et Physique. 10: 113-121(1838)

9. A. Bonasera and H. Zheng, https://www.medrxiv.org/lookup/doi/10.1101/2020.05.07.20094235 